\title{
Aldehydes as Photoremovable Directing Groups: Synthesis of Pyrazoles by a Photocatalyzed [3+2] Cycloaddition/Norrish Type Fragmentation Sequence
}

\author{
Ana Pascual-Escudero, Laura Ortiz-Rojano, Silvia Simón-Fuente, Javier Adrio,* and María Ribagorda*
}

Cite This: Org. Lett. 2021, 23, 4903-4908

Read Online

\section{ACCESS}

Џll Metrics \& More

回国 Article Recommendations

Supporting Information

ABSTRACT: A straightforward methodology for the regioselective synthesis of pyrazoles has been developed by a domino sequence based on a photoclick cycloaddition followed by a photocatalyzed oxidative deformylation reaction. Distinguishing features of this protocol include an unprecedented photoredox-catalyzed Norrish type fragmentation under green-light irradiation and the use of $\alpha, \beta$-unsaturated aldehydes as synthetic equivalents of alkynes, where the aldehyde is acting as a novel photoremovable directing group.

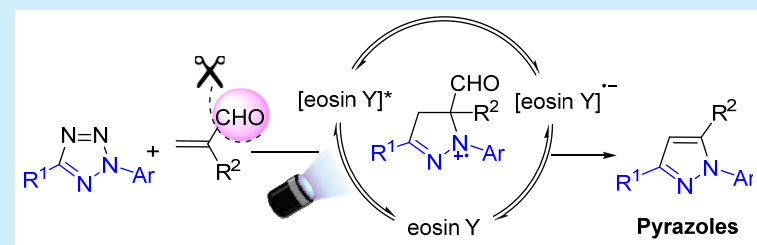

Photoinduced 1,3-dipolar cycloaddition/oxidative deformylation
$\mathrm{P}$ yrazoles are privileged structures in organic and medicinal chemistry, because they are present in numerous natural products and therapeutic agents based on small molecules, such as Celecobix, Rimonabant, or Lersivirine, among others. ${ }^{1}$ Furthermore, pyrazoles have been extensively used as ligands in transition metal-catalyzed processes. ${ }^{2}$ Accordingly, the development of new methods to facilitate the concise preparation of structurally diverse pyrazoles is a very appealing synthetic goal. Among the plethora of reported methodologies for pyrazole synthesis, the traditional condensation of hydrazine derivatives with diverse substituted carbonyl compounds has been the most commonly employed. ${ }^{3}$ Alternatively, 1,3-dipolar cycloaddition has also played a prevalent role due to its great versatility. ${ }^{4}$ The use of diazoalkanes $^{5}$ or nitrile imines ${ }^{6}$ as dipoles and activated alkynes as dipolarophiles is a straightforward procedure for the preparation of pyrazoles. With activated alkenes as dipolarophiles, the resulting pyrazolines required an extra elimination ${ }^{7}$ or oxidation step ${ }^{8}$ for aromatization to synthesize pyrazoles. However, non-activated alkyl-substituted alkynes or alkenes have been scarcely studied because they usually lead to regioisomeric mixtures in low conversions.

Traditionally, the nitrile imine dipoles can be generated in situ either from $\alpha$-halohydrazones in the presence of a base ${ }^{9}$ or from tetrazole precursors. Of special relevance is the lightinduced 1,3-dipolar cycloadditions of 1,3-diaryltetrazoles that have been widely applied in biological and material chemistry (Scheme 1). ${ }^{10}$ Huisgen and co-workers ${ }^{11}$ reported the first example of this photoactivated cycloaddition for the synthesis of pyrazolines. More recently, Lin and co-workers ${ }^{12}$ have proven the usefulness of this clean and atom-economical transformation with a wide range of alkenes as dipolarophiles (Scheme 1, pyrazolines).
Scheme 1. Synthesis of Pyrazoles and Pyrazolines from Tetrazoles

Previous work: Tetrazole alkyne/alkene photoinduced cycloaddition

$$
\begin{aligned}
& \overbrace{N^{-}}^{N}=-N_{-} \\
& \frac{\mathrm{h} v(\mathrm{UV}), \bar{\equiv} \mathrm{R}^{1}}{\mathrm{R}^{1}=\mathrm{CO}_{2} \mathrm{R}, \mathrm{Ar}}
\end{aligned}
$$

$\mathrm{hv}(\mathrm{UV}) \bigvee \mathrm{N}_{2}$
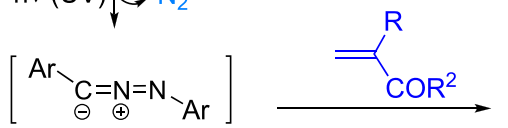

variable mixture of regioisomers

nitrile imines

$\mathrm{R}^{2}=\mathrm{H}, \mathrm{Me}, \mathrm{OR}, \mathrm{NR}_{2}$<smiles>[R]OC1CC([Al])=NN1[Tl]</smiles>

This work: Photoinduced cycloaddition/Eosin based photocatalytic

$$
\text { Norrish type fragmentation }
$$

$\alpha, \beta$-Unsaturated aldehydes have been extensively used as dipolarophiles in 1,3-dipolar cycloadditions ${ }^{13}$ with a great variety of dipoles. ${ }^{14,15}$ The synthetic versatility of the formyl group is beyond any doubt because it can be straightforwardly converted into many other functional groups. However, examples of reactions involving $\mathrm{C}-\mathrm{C}$ bond cleavage by formyl

Received: May 17, 2021

Published: June 7, 2021 
group elimination are scarce. Conversely, the photochemical UV-induced cleavage of aldehydes or ketones into two radical intermediates, known as the Norrish reaction, has been widely studied, including several applications in natural product synthesis. ${ }^{16}$ Because there are not reported procedures for the efficient utilization of non-activated alkynes as dipolarophiles in this kind of cycloaddition, we envisaged that an $\alpha, \beta$ unsaturated aldehyde could act as an alkyne surrogate, increasing the reactivity and temporally controlling the regioselectivity of the cycloaddition. Herein, we report a sequential dipolar photoclick reaction followed by a Norrish type deformylation step under green-light irradiation using eosin $\mathrm{Y}$ as a photoredox catalyst. To the best of our knowledge, there are no photocatalytic examples for this fragmentation besides the important advantages of this approximation. ${ }^{17,18}$

In connection with our previous work on tetrazole photoclick 1,3-dipolar cycloadditions, ${ }^{19}$ we set out to explore the application of this methodology to the synthesis of pyrazoles using unsaturated aldehydes as synthetic equivalents of alkynes.

For this purpose, we initially studied the Norrish type deformylation reaction of formyl pyrazoline 1a, previously prepared by a UV-light-induced dipolar cycloaddition reaction between the corresponding diaryltetrazole and methacrolein. ${ }^{20}$ UV-light irradiation of 1a gave the desired pyrazole $2 \mathrm{a}$ in $44 \%$ yield after $24 \mathrm{~h}$ (Table 1, entry 1 ). This result evidenced the potential use of methacrolein as a synthetic equivalent of propyne gas leading to a regioselective synthesis of pyrazole $\mathbf{2 a}$. Our next purpose was to evaluate the $\mathrm{C}-\mathrm{C}$ fragmentation process using a photocatalyst under irradiation with a less

Table 1. Optimization of the Reaction Conditions

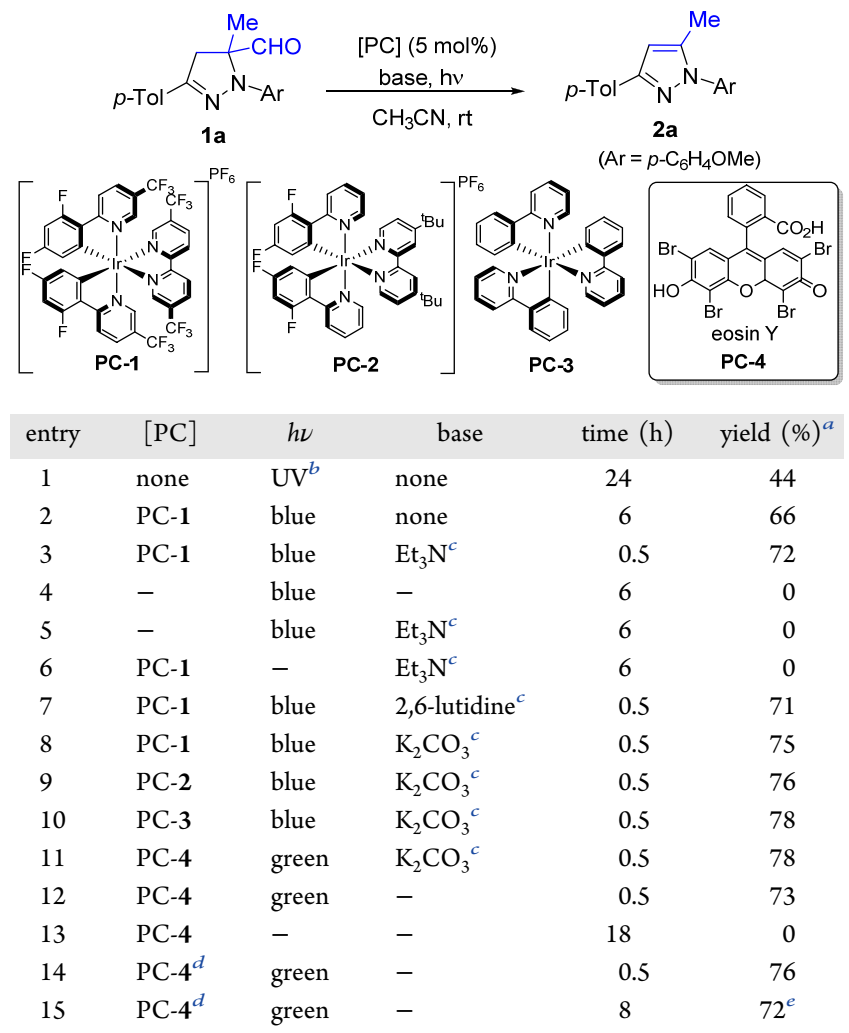

${ }^{a}$ Isolated yield after purification. ${ }^{b} \mathrm{UV} \lambda 315 \mathrm{~nm} .{ }^{c}$ With 1.5 equiv. ${ }^{d}$ With $1 \mathrm{~mol} \%$ eosin Y. ${ }^{e} \mathrm{On}$ a $3.5 \mathrm{mmol}$ scale (gram-scale). Blue LEDs at a $\lambda$ of $420 \mathrm{~nm}$ and green LEDs at a $\lambda$ of $535 \mathrm{~nm}$. energetic light. We were pleased to find that iridium PC-1 (5 mol \%) was able to catalyze the oxidative deformylation reaction under blue light $(420 \mathrm{~nm})$, affording $2 \mathrm{a}$ in $66 \%$ yield after $6 \mathrm{~h}$ (Table 1 , entry 2 ). The addition of $\mathrm{Et}_{3} \mathrm{~N}$ significantly increased the reaction rate, allowing the isolation of $2 \mathrm{a}$ in $72 \%$ yield after $30 \mathrm{~min}$ (entry 3 ). Control experiments showed that light irradiation and a photocatalyst were indispensable for the reaction to proceed (entries 4-6). The use of other bases such as 2,6-lutidine or $\mathrm{K}_{2} \mathrm{CO}_{3}$ gave similar results, although cleaner reaction mixtures were obtained in the latter case (entries 7 and 8). Other iridium sources such as PC-2 or PC-3 successfully worked (entries 9 and 10). Finally, the reaction using inexpensive eosin $\mathrm{Y}(5 \mathrm{~mol} \%)$, as an organic photocatalyst, under green LED irradiation provided $\mathbf{2 a}$ in $78 \%$ yield (entry 11 ). The reaction without the base displayed a similar efficiency (entry 12). A control experiment proved that green-light irradiation was necessary for the reaction to take place (entry 13). The organic catalyst loading could be decreased to $1 \mathrm{~mol} \%$ without impairing the reaction yield (entry 14). The transformation was also successfully performed on a gram scale (entry 15 ).

With the optimized reaction conditions in hand, we wondered if this pyrazole scaffold could be accessed from 1,3-diaryltetrazoles in a domino process that would embrace a photoclick dipolar cycloaddition followed by an oxidative deformylation. For this purpose, an Ultra-Vitalux (OSRAM) lamp ${ }^{20}$ was used as the irradiation source. This lamp has emission signals at $\lambda$ values of $315 \mathrm{~nm}$ (UV), $440 \mathrm{~nm}$ (blue), and $540 \mathrm{~nm}$ (green), which would allow both UV-lightpromoted cycloaddition and the blue- or green-light-photocatalyzed deformylation process, providing an easier reaction setup. $^{21}$ Gratifyingly, the reaction between tetrazole $3 a$ and methacrolein gave $2 \mathrm{a}$ as a single regioisomer in $72 \%$ yield, after $5 \mathrm{~h}$ at $\mathrm{rt}$ (Scheme 2).

Scheme 2. Domino Photoinduced Cycloaddition/Oxidative Deformylation Sequence

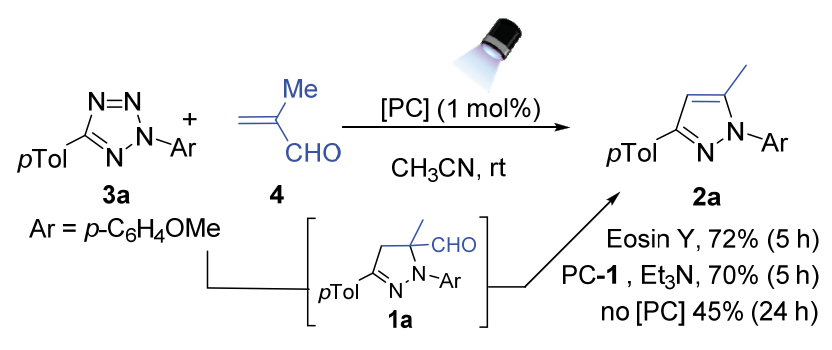

The process using $\mathrm{Ir}-\mathrm{PC}-\mathbf{1}$ and $\mathrm{Et}_{3} \mathrm{~N}$ also gave $\mathbf{2 a}$ in a $70 \%$ yield. The use of blue or green LEDs as the single irradiation source resulted in the full recovery of tetrazole 3a, underlining that the photoredox catalyst was exclusively catalyzing the second step. The reaction without any photocatalyst under the Ultra-Vitalux lamp gave pyrazole $\mathbf{2 a}$ in significantly lower yield and longer reaction times (irradiation for $24 \mathrm{~h}$ ) (Scheme 2). The global process using the photoredox catalyst opens the possibility of using the formyl group as novel photoremovable directing groups under mild reaction conditions. ${ }^{22}$

The simple one-step preparation of pyrazoles led us to study the scope of the process with regard to the substitution at the nitrile imine precursor. As shown in Scheme 3, different electron-donating and electron-withdrawing groups underwent the dipolar/oxidative deformylation process successfully. $o$-, 
Scheme 3. Scope of the Domino Sequence
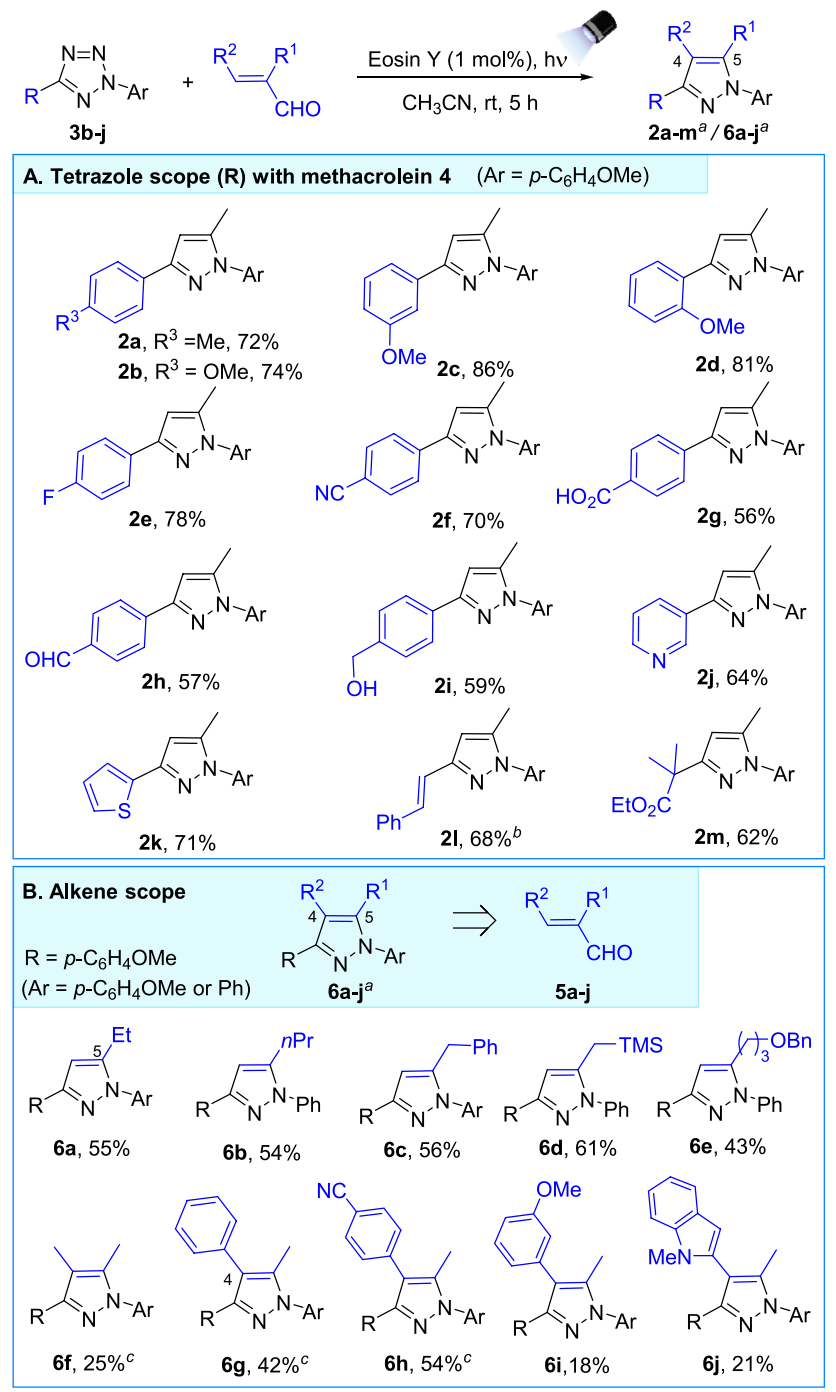

${ }^{a}$ Isolated yield. ${ }^{b}$ Eosin Y (5 mol \%). ${ }^{c}$ Eosin Y (2 mol \%).

$m$-, and $p$-methoxy-substituted phenyl tetrazoles furnished the corresponding pyrazoles in good yields $(\mathbf{2} \mathbf{b}-\mathbf{d}$, respectively). Different functional groups such as fluoro, cyano, or carboxylic acids were perfectly compatible $(\mathbf{2} \mathbf{e}-\mathbf{g}$, respectively). Interestingly, because no external oxidant was required in this protocol, groups sensitive to oxidant conditions such as formyl or benzylic alcohol were well tolerated ( $2 \mathbf{h}$ and $\mathbf{2 i}$ ). Similarly, 3-heteroaromatic substituted pyrazoles, such as pyridyl or thienyl derivative $\mathbf{2} \mathbf{j}$ or $\mathbf{2} \mathbf{k}$, respectively, were also obtained in good yields. The cycloaddition of tetrazole 31 bearing an alkenyl substituent proceeded similarly, giving rise to $\mathbf{2 l}$ as the only detectable isomer (no isomerization of the remaining double bond was observed). ${ }^{23}$ Alkyl-substituted pyrazole $2 \mathbf{m}$ was also obtained in good yield.

Next, we studied the compatibility of this photocatalyzed cycloaddition/fragmentation sequence with other $\alpha, \beta$-unsaturated aldehydes as dipolarophiles (Scheme $3 \mathrm{~B}$ ). The reaction of 2-ethyl and 2-propyl acrolein under the optimized reaction conditions afforded pyrazoles $\mathbf{6 a}$ and $\mathbf{6 b}$ in $55 \%$ and $54 \%$ yields, respectively. A benzyl substituent in the aldehyde was also well tolerated, leading to pyrazole $6 \mathrm{c}$ in $56 \%$ yield. The reaction sequence proceeded in similar yields with function- alized alkyl groups at the $\alpha$-position of the aldehyde (pyrazoles 6d and $6 \mathrm{e}$ ).

To evaluate the scope of this reaction with more sterically challenging substrates, $\alpha, \beta$-disubstituted aldehydes were next evaluated (Scheme 3B). Despite a decrease in the reactivity observed in the first dipolar cycloaddition step, pyrazoles $\mathbf{6 f}-\mathbf{j}$ could be obtained as single regioisomers. All of these results pointed out that this protocol opens a regioselective access to 5-alkyl tri- and tetrasubtituted pyrazoles, which was elusive with other cycloaddition methodologies. ${ }^{5-8}$

It is known that ketones are also suitable substrates for Norrish fragmentation reactions. Therefore, we next studied the possibility of expanding the scope of this methodology to the use of $\alpha, \beta$-unsaturated methyl ketones as dipolarophiles. For this purpose, we prepared pyrazoline $\mathbf{1 b}$, with a pendant methyl ketone, through the corresponding 1,3-dipolar cycloaddition between $3 \mathbf{a}$ and 3-methyl-3-buten-2-one. The reaction of $\mathbf{1 b}$ under optimized reaction conditions was completed after $1 \mathrm{~h}$, affording pyrazole 2 a together with compound 7 , in a $54: 46$ ratio (Scheme $4 \mathrm{~A}$ ). Although in this case pyrazole $2 \mathrm{a}$

Scheme 4. Photocatalyzed Fragmentation of $1 \mathrm{~b}, 9$, and 1a

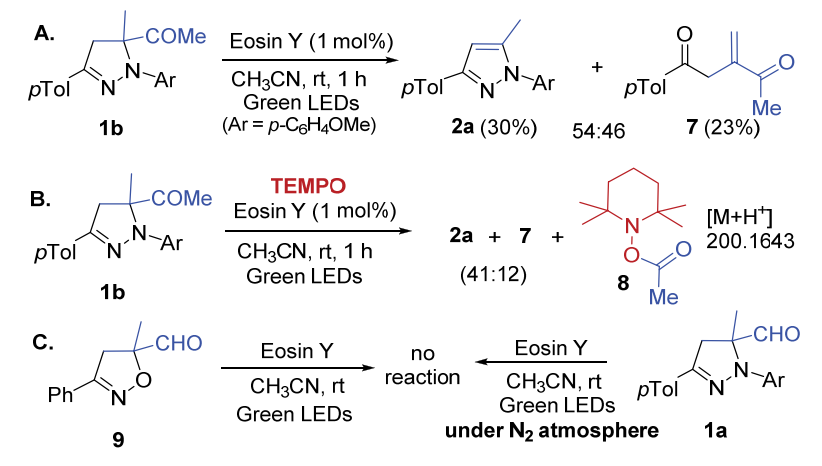

was obtained in moderate yield, this result highlights the significant potential of this green-light-catalyzed $\mathrm{C}-\mathrm{C}$ fragmentation. The reaction with other 2-methyl $\alpha, \beta$ unsaturated carbonyl derivatives, such as the carboxylic acid, the ethyl ester, or the $N$-methyl amide, gave the corresponding pyrazolines from the dipolar reaction; however, no carbonyl fragmentation was observed in any case. ${ }^{24}$

To improve our understanding of the reaction pathway, we conducted a series of experiments. The addition of radical scavenger TEMPO $[(2,2,6,6$-tetramethylpiperidin-1-yl)oxyl $]$ to the photoreaction of $\mathbf{1} \mathbf{b}$ slowed the fragmentation process. As a result, a mixture of starting pyrazoline $\mathbf{1 b}$, pyrazole $\mathbf{2 a}$, and compound 7 was observed in a 47:41:12 ratio after irradiation for $1 \mathrm{~h}$ (detected by ${ }^{1} \mathrm{H}$ NMR). This reaction was also monitored by ESI-MS detecting the $\left[\mathrm{M}+\mathrm{H}^{+}\right]$ion $(\mathrm{m} / z$ 200.1643) that corresponds to TEMPO adduct 8, which indicates the involvement of the acyl radical (Scheme 4B). ${ }^{20,25}$ Moreover, the reaction did not proceed with formyl dihydroisoxazole 9, recovering the starting material unaltered. This is suggestive of the involvement of $\mathrm{N}-1$ of the pyrazoline under the photoredox process. We also observed that the reaction was completely inhibited in the absence of $\mathrm{O}_{2}$ (reaction under $\mathrm{N}_{2}$ through a "freeze-pump-thaw" cycle) (Scheme 4C).

On the basis of all of these results, a plausible mechanism is proposed in Scheme 5. First, photolysis of tetrazole 3 generates nitrile imine dipole that, upon 1,3-dipolar cycloaddition with 
Scheme 5. Mechanistic Proposal for the Domino Photoinduced Cycloaddition/Oxidative Deformylation

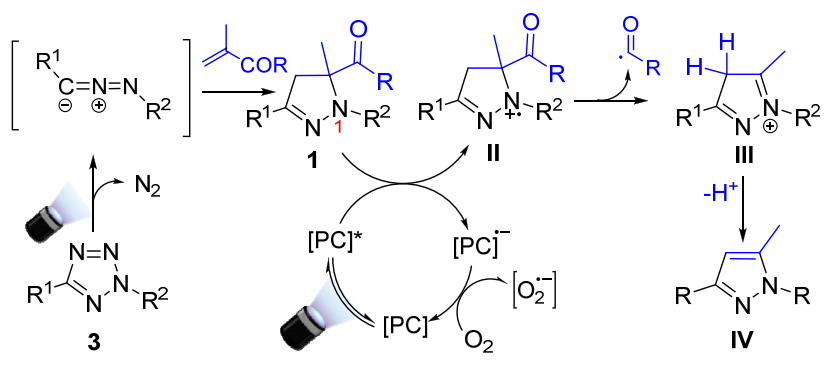

the $\alpha, \beta$-unsaturated compound, leads to pyrazoline 1 . Visiblelight irradiation of eosin $\mathrm{Y}$ generates an excited-state oxidant $\left(E_{1 / 2}=0.86 \mathrm{~V}\right.$ vs SCE $)$ that can accept a single electron transfer from N-1 of pyrazoline (for $1 \mathrm{a}, E_{1 / 2}=0.68 \mathrm{~V}$ vs SCE) ${ }^{20}$ thus forming aminyl radical cation II and the reduced state of the photocatalyst. ${ }^{26}$ Radical cation II evolves via a C$\mathrm{C}$ bond fragmentation of the carbonyl group, generating a formyl $(\mathrm{R}=\mathrm{H})$ or an acyl radical $(\mathrm{R}=\mathrm{Me})$ favored by the formation of pyrazolinium cation III that aromatizes to the corresponding pyrazole IV after losing a proton. Oxidation of the photocatalyst by oxygen completes the catalytic cycle. The lack of reactivity under a nitrogen atmosphere supports the proposed reductive quenching cycle. Moreover, the formation of byproduct 7 (Scheme 4A) in the reaction with 3-methyl-3buten-2-one supported the formation of radical cation II ( $\mathrm{R}=$ $\mathrm{Me}$ ), which can evolve via $\beta$-hydrogen abstraction of the methyl group, followed by a ring opening of the heterocycle. The formation of $\mathbf{2 a}$ without a photocatalyst (Table 1, entry 1 ) and under UV light could be rationalized on the basis of a similar photoclick reaction followed in this case by a UV-light Norrish type I formyl fragmentation ${ }^{16}$ and oxidation sequence.

In conclusion, an innovative procedure for the preparation of pyrazoles has been developed using a domino photoinduced 1,3-dipolar cycloaddition/photoredox-catalyzed formyl fragmentation sequence. To the best of our knowledge, this is the first report describing an oxidative deformylation reaction using a photoredox catalyst under visible-light irradiation. An important advantage is that the domino sequence took place in the presence of an inexpensive organic photocatalyst such as eosin Y. The regiocontrol exerted by the formyl group allowed better access to tri- and tetrasubstituted 5-alkyl pyrazoles. Moreover, this protocol is compatible with the presence of groups sensitive to oxidizing conditions. These results demonstrate the possibility of using aldehydes as a clean photoremovable directing group and open the door for further investigations of other different systems.

\section{ASSOCIATED CONTENT}

\section{SI Supporting Information}

The Supporting Information is available free of charge at https://pubs.acs.org/doi/10.1021/acs.orglett.1c01665.

General experimental procedures, characterization data, and NMR spectra (PDF)

\section{AUTHOR INFORMATION}

\section{Corresponding Authors}

María Ribagorda - Departamento de Química Orgánica, Facultad de Ciencias, Universidad Autónoma de Madrid,
28049 Madrid, Spain; Institute for Advanced Research in Chemical Sciences (IAdChem), Universidad Autónoma de Madrid, 28049 Madrid, Spain; (1) orcid.org/0000-0001-

7185-4095; Email: maria.ribagorda@uam.es

Javier Adrio - Departamento de Química Orgánica, Facultad de Ciencias, Universidad Autónoma de Madrid, 28049 Madrid, Spain; Institute for Advanced Research in Chemical Sciences (IAdChem), Universidad Autónoma de Madrid, 28049 Madrid, Spain; 이이.org/0000-0001-6238-6533; Email: javier.adrio@uam.es

\section{Authors}

Ana Pascual-Escudero - Departamento de Química Orgánica, Facultad de Ciencias, Universidad Autónoma de Madrid, 28049 Madrid, Spain; () orcid.org/0000-00015780-1739

Laura Ortiz-Rojano - Departamento de Química Orgánica, Facultad de Ciencias, Universidad Autónoma de Madrid, 28049 Madrid, Spain

Silvia Simón-Fuente - Departamento de Química Orgánica, Facultad de Ciencias, Universidad Autónoma de Madrid, 28049 Madrid, Spain

Complete contact information is available at:

https://pubs.acs.org/10.1021/acs.orglett.1c01665

\section{Notes}

The authors declare no competing financial interest.

\section{ACKNOWLEDGMENTS}

This work was supported by the MINECO (CTQ2014-53894$\mathrm{R}$ and CTQ2017-85454-C2-2-P) and FEDER/MICIU (PGC2018-098660-B-I00) of Spain. L.O.-R. thanks MINECO for a FPI fellowship, and A.P.-E. thanks CAM for a postdoctoral fellowship.

\section{DEDICATION}

This paper is dedicated to Professor Carmen Carreño on the occasion of her retirement from Universidad Autonoma de Madrid.

\section{REFERENCES}

(1) (a) Elguero, J.; Silva, A. M. S.; Tomé, A. C. In Modern Heterocyclic Chemistry; Alvarez-Builla, J., Vaquero, J. J., Barluenga, J., Eds.; Wiley-VCH: Weinheim, Germany, 2011; Vol. 2, pp 635-725. (b) Karrouchi, K.; Radi, S.; Ramli, Y.; Taoufik, J.; Mabkhot, Y. N.; AlAizari, F. A.; Ansar, M. Synthesis and Pharmacological Activities of Pyrazole Derivatives: A Review. Molecules 2018, 23, 134. (c) Ansari, A.; Ali, A.; Asif, M.; Shamsuzzaman, S. Review: biologically active pyrazole derivatives. New J. Chem. 2017, 41, 16. (d) Khan, M. F.; Alam, M. M.; Verma, G.; Akhtar, W.; Akhter, M.; Shaquiquzzaman, M. The therapeutic voyage of pyrazole and its analogs: A review. Eur. J. Med. Chem. 2016, 120, 170.

(2) (a) Mukherjee, R. Coordination chemistry with pyrazole-based chelating ligands: molecular structural aspects. Coord. Chem. Rev. 2000, 203, 151. (b) Keter, F. K.; Darkwa, J. Perspective: the potential of pyrazole-based compounds in medicine. BioMetals 2012, $25,9$. (c) Bellarosa, L.; Diez, J.; Gimeno, J.; Lledos, A.; Suarez, F. J.; Ujaque, G.; Vicent, C. Highly Efficient Redox Isomerisation of Allylic Alcohols Catalysed by Pyrazole-Based Ruthenium(IV) Complexes in Water: Mechanisms of Bifunctional Catalysis in Water. Chem. - Eur. J. 2012, $18,7749$.

(3) (a) Brown, A. W. Recent Developments in the Chemistry of Pyrazoles. Adv. Heterocycl. Chem. 2018, 126, 55. (b) Fustero, S.; Sánchez-Roselló, M.; Barrio, P.; Simón-Fuentes, A. From 2000 to 
Mid-2010: A Fruitful Decade for the Synthesis of Pyrazoles. Chem. Rev. 2011, 111, 6984. (c) Gonzalez-Ortega, A.; Luisa Sadaba, M.; Alberola, A.; Calvo Bleye, L.; Carmen Sanudo, M. Scope and Limitations in the Regioselective Synthesis of 1,3,5-Trisubstituted Pyrazoles from $\beta$-Amino Enones and Hydrazine Derivatives. ${ }^{13} \mathrm{C}$ Chemical Shift Prediction Rules for 1,3,5-Trisubstituted Pyrazoles. Heterocycles 2001, 55, 331. (d) Stauffer, S. R.; Huang, Y.; Coletta, C. J.; Tedesco, R.; Katzenellenbogen, J. A. Estrogen pyrazoles: defining the pyrazole core structure and the orientation of substituents in the ligand binding pocket of the estrogen receptor. Bioorg. Med. Chem. 2001, 9, 141. (e) Ding, Y.; Zhang, T.; Chen, Q.-Y.; Zhu, C. VisibleLight Photocatalytic Aerobic Annulation for the Green Synthesis of Pyrazoles. Org. Lett. 2016, 18, 4206.

(4) (a) Hashimoto, T.; Maruoka, K. Recent Advances of Catalytic Asymmetric 1,3-Dipolar Cycloadditions. Chem. Rev. 2015, 115, 5366. (b) Padwa, A.; Pearson, W. H. In Synthetic Applications of 1,3-Dipolar Cycloaddition Chemistry toward Heterocycles and Natural Products; Wiley: Hoboken, NJ, 2003.

(5) (a) Aggarwal, V. K.; de Vicente, J.; Bonnert, R. V. A Novel OnePot Method for the Preparation of Pyrazoles by 1,3-Dipolar Cycloadditions of Diazo Compounds Generated in Situ. J. Org. Chem. 2003, 68, 5381. (b) Hari, Y.; Tsuchida, S.; Sone, R.; Aoyama, T. An Efficient Synthesis of 2-Diazo-2-(trimethylsilyl)ethanols and Their Application to Pyrazole Synthesis. Synthesis 2007, 2007, 3371. (c) Pérez-Aguilar, M. C.; Valdes, C. Synthesis of Chiral Pyrazoles: A 1,3-Dipolar Cycloaddition/[1,5] Sigmatropic Rearrangement with Stereoretentive Migration of a Stereogenic Group. Angew. Chem., Int. Ed. 2015, 54, 13729. (d) Zheng, Y.; Zhang, X.; Yao, R.; Wen, Y.; Huang, J.; Xu, X. 1,3-Dipolar Cycloaddition of Alkyne-Tethered NTosylhydrazones: Synthesis of Fused Polycyclic Pyrazoles. J. Org. Chem. 2016, 81, 11072. (e) Li, F.; Nie, J.; Sun, L.; Zheng, Y.; Ma, J.-A. Silver-Mediated Cycloaddition of Alkynes with $\mathrm{CF}_{3} \mathrm{CHN}_{2}$ : Highly Regioselective Synthesis of 3-Trifluoromethylpyrazoles. Angew. Chem., Int. Ed. 2013, 52, 6255.

(6) (a) Remy, R.; Bochet, C. G. Application of Photoclick Chemistry for the Synthesis of Pyrazoles via 1,3-Dipolar Cycloaddition between Alkynes and Nitrilimines Generated In Situ. Eur. J. Org. Chem. 2018, 2018, 316. (b) Voronin, V. V.; Ledovskaya, M. S.; Gordeev, E. G.; Rodygin, K. S.; Ananikov, V. P. $[3+2]$-Cycloaddition of in Situ Generated Nitrile Imines and Acetylene for Assembling of 1,3Disubstituted Pyrazoles with Quantitative Deuterium Labeling. J. Org. Chem. 2018, 83, 3819.

(7) (a) Xie, J.-W.; Wang, Z.; Yang, W.-J.; Kong, L.-C.; Xu, D.-C. Efficient method for the synthesis of functionalized pyrazoles by catalyst-free one-pot tandem reaction of nitroalkenes with ethyl diazoacetate. Org. Biomol. Chem. 2009, 7, 4352. (b) Tang, M.; Zhang, W.; Kong, Y. DABCO-promoted synthesis of pyrazoles from tosylhydrazones and nitroalkenes. Org. Biomol. Chem. 2013, 11, 6250.

(8) (a) Padwa, A.; Nahm, S.; Sato, E. Photochemical transformations of small ring heterocyclic compounds. 9. Intramolecular 1,3-dipolar cycloaddition reactions of alkenyl-subituted nitrile imines. J. Org. Chem. 1978, 43, 1664. (b) Pal, G.; Paul, S.; Ghosh, P. P.; Das, A. R. $\mathrm{PhIO}$ promoted synthesis of nitrile imines and nitrile oxides within a micellar core in aqueous media: a regiocontrolled approach to synthesizing densely functionalized pyrazole and isoxazoline derivatives. RSC Adv. 2014, 4, 8300. (c) Deng, X.; Mani, N. S. Reaction of N-Monosubstituted Hydrazones with Nitroolefins: A Novel Regioselective Pyrazole Synthesis. Org. Lett. 2006, 8, 3505. (d) Shao, Y.; Tong, J.; Zhao, Y.; Zheng, H.; Ma, L.; Ma, M.; Wan, X. [3 + 2] cycloaddition and subsequent oxidative dehydrogenation between alkenes and diazo compounds: a simple and direct approach to pyrazoles using TBAI/TBHP. Org. Biomol. Chem. 2016, 14, 8486.

(9) (a) Huisgen, R.; Seidel, M.; Wallbillich, G.; Knupfer, H. Diphenyl-nitrilimin und seine 1.3-dipolaren additionen an alkene und alkine. Tetrahedron 1962, 17, 3. (b) Meazza, G.; Zanardi, G.; Piccardi, P. J. A convenient and versatile synthesis of 4-trifluoromethylsubstituted pyrazoles. J. Heterocycl. Chem. 1993, 30, 365. (c) Bonini, B. F.; Franchini, M. C.; Gentili, D.; Locatelli, E.; Ricci, A. 1,3-Dipolar Cycloaddition of Nitrile Imines with Functionalized Acetylenes:
Regiocontrolled $\mathrm{Sc}(\mathrm{OTf})_{3}$-Catalyzed Synthesis of 4- and 5-Substituted Pyrazoles. Synlett 2009, 2009, 2328.

(10) (a) Ramil, C. P.; Lin, Q. Photoclick chemistry: a fluorogenic light-triggered in vivo ligation reaction. Curr. Opin. Chem. Biol. 2014, 21, 89. (b) Lederhose, P.; Abt, D.; Welle, A.; Müller, R.; BarnerKowollik, C.; Blinco, J. P. Exploiting $\lambda$-Orthogonal Photoligation for Layered Surface Patterning. Chem. - Eur. J. 2018, 24, 576. (c) Schart, V. F.; Hassenrück, J.; Späte, A.-K.; Dold, J. E. G. A.; Fahrner, R.; Wittmann, V. Triple Orthogonal Labeling of Glycans by Applying Photoclick Chemistry. ChemBioChem 2019, 20, 166.

(11) Clovis, J. S.; Eckell, A.; Huisgen, R.; Sustmann, R. 1.3-Dipolare Cycloadditionen, XXV. Der Nachweis des freien Diphenylnitrilimins als Zwischenstufe bei Cycloadditionen. Chem. Ber. 1967, 100, 60.

(12) (a) Wang, Y.; Rivera Vera, C. I.; Lin, Q. Convenient Synthesis of Highly Functionalized Pyrazolines via Mild, Photoactivated 1,3Dipolar Cycloaddition. Org. Lett. 2007, 9, 4155. (b) Wang, Y.; Song, W.; Hu, W. J.; Lin, Q. Fast alkene functionalization in vivo by Photoclick chemistry: HOMO lifting of nitrile imine dipoles. Angew. Chem., Int. Ed. 2009, 48, 5330. (c) An, P.; Lin, Q. Sterically shielded tetrazoles for a fluorogenic photoclick reaction: tuning cycloaddition rate and product fluorescence. Org. Biomol. Chem. 2018, 16, 5241.

(13) Keiko, N. A.; Vchislo, N. V. Synthesis of Diheteroatomic FiveMembered Heterocyclic Compounds from $\alpha, \beta$-Unsaturated Aldehydes. Asian J. Org. Chem. 2016, 5, 1169.

(14) For references using different dipoles, see: (a) Bădoiu, A.; Kündig, E. P. Electronic effects in 1,3-dipolar cycloaddition reactions of N-alkyl and N-benzyl nitrones with dipolarophiles. Org. Biomol. Chem. 2012, 10, 114. (b) Brinkmann, Y.; Madhushaw, R. J.; Jazzar, R.; Bernardinelli, G.; Kündig, E. P. Chiral ruthenium Lewis acid-catalyzed nitrile oxide cycloadditions. Tetrahedron 2007, 63, 8413. (c) Li, W.; Jia, Q.; Du, Z.; Wang, J. Direct access to triazole-olefins through catalytic cycloaddition of azides to unsaturated aldehydes. Chem. Commun. 2013, 49, 10187. (d) Chen, W.; Yuan, X.-H.; Li, R.; Du, W.; Wu, Y.; Ding, L.-S.; Chen, Y.-C. Organocatalytic and Stereoselective $[3+2]$ Cycloadditions of Azomethine Imines with $\alpha, \beta$-Unsaturated Aldehydes. Adv. Synth. Catal. 2006, 348, 1818. (e) Cabrera, S.; Gómez Arrayás, R.; Carretero, J. C. Highly Enantioselective Copper(I)-Fesulphos-Catalyzed 1,3-Dipolar Cycloaddition of Azomethine Ylides. J. Am. Chem. Soc. 2005, 127, 16394.

(15) (a) Kano, T.; Hashimoto, T.; Maruoka, K. Enantioselective 1,3Dipolar Cycloaddition Reaction between Diazoacetates and $\alpha$ Substituted Acroleins: Total Synthesis of Manzacidin A. J. Am. Chem. Soc. 2006, 128, 2174. (b) Gao, L.; Hwang, G.-S.; Lee, M. Y.; Ryu, D. H. Catalytic enantioselective 1,3-dipolar cycloadditions of alkyldiazoacetates with $\alpha, \beta$-disubstituted acroleins. Chem. Commun. 2009, 5460 .

(16) (a) Norrish, R. G. W.; Bamford, C. H. Photodecomposition of Aldehydes and Ketones. Nature 1936, 138, 1016. (b) Miranda, M. A.; Font-Sanchis, E.; Pérez-Prieto, J. Photochemistry of Acyl-Alkyl Biradicals. J. Org. Chem. 1999, 64, 3802. (c) Dake, G. R.; Fenster, E. E.; Patrick, B. O. A Synthetic Approach to the Fusicoccane A-B Ring Fragment Based on a Pauson-Khand Cycloaddition/Norrish Type 1 Fragmentation. J. Org. Chem. 2008, 73, 6711. (d) Horspool, W. M. In Photochemistry: Photolysis of carbonyl compounds; Dunkin, I., Ed.; Royal Society of Chemistry: London, 2005; Vol. 35, pp 1-16. (e) Karkas, M. D.; Porco, J. A.; Stephenson, C. R. J. Photochemical Approaches to Complex Chemotypes: Applications in Natural Product Synthesis. Chem. Rev. 2016, 116, 9683.

(17) (a) Chu, L.; Lipshultz, J. M.; MacMillan, D. W. C. Merging Photoredox and Nickel Catalysis: The Direct Synthesis of Ketones by the Decarboxylative Arylation of $\alpha$-OxoAcids. Angew. Chem., Int. Ed. 2015, 54, 7929. (b) Stephenson, C. R. J., Yoon, T. P., MacMillan, D. W. C., Eds. Visible Light Photocatalysis in Organic Chemistry; WileyVCH Verlag: Weinheim, Germany, 2018. (c) For a cerium photocatalytic formal $\mathrm{C}-\mathrm{C}$ bond cleavage of tertiary alcohols from ketones, see: Chen, Y.; Du, J.; Zuo, Z. Selective C-C Bond Scission of Ketones via Visible-Light-Mediated Cerium Catalysis. Chem. 2020, 6, 266. 
(18) For visible-light-mediated Norrish chemistry of $\alpha$-carbonyl ketones, see: (a) Roque, J. B.; Kuroda, Y.; Jurczyk, J.; Xu, L.-P.; Ham, J. S.; Göttemann, L. T.; Roberts, C. A.; Adpressa, D.; Saurí, J.; Joyce, L. A.; Musaev, D. G.; Yeung, C. S.; Sarpong, R. C-C Cleavage Approach to $\mathrm{C}-\mathrm{H}$ Functionalization of Saturated Aza-Cycles Roque. ACS Catal. 2020, 10, 2929-2941. (b) Alvarez-Dorta, D.; León, E. I.; Kennedy, A. R.; Martín, A.; Pérez-Martín, I.; Riesco-Fagundo, C.; Suárez, E. Sequential Norrish Type II Photoelimination and Intramolecular Aldol Cyclization of $\alpha$-Diketones: Synthesis of Polyhydroxylated Cyclopentitols by Ring Contraction of Hexopyranose Carbohydrate Derivatives. Chem. - Eur. J. 2013, 19, 1031210333.

(19) Ortiz-Rojano, L.; Rojas-Martin, J.; Rodriguez-Diaz, C.; Carreño, M. C.; Ribagorda, M. Light-Induced Tetrazole-Quinone 1,3-Dipolar Cycloadditions. Chem. - Eur. J. 2019, 25, 15050.

(20) For details, see the Supporting Information.

(21) Alternatively, a UV lamp combined with green or blue LEDs can be use as the light irradiation source.

(22) For the synthesis of 5-alkyl-1H-pyrazolines by a formal deformylation, see: Chen, Y.-C.; Zhu, M.-K.; Loh, T.-P Csp ${ }^{3}-\mathrm{Csp}^{3}$ Bond Cleavage in the Palladium-Catalyzed Aminohydroxylation of Allylic Hydrazones Using Atmospheric Oxygen as the Sole Oxidant. Org. Lett. 2015, 17, 2712-2715.

(23) The reaction between 31 and methacrolein in the absence of eosin gave a 1:1 mixture of E-2l and Z-2l isomers, probably as a consequence of the long irradiation times required to complete the reaction ( 1 day).

(24) For other $\alpha, \beta$-unsaturated aldehydes and ketones that were not suitable for this study as dipolarophiles, see the Supporting Information.

(25) All of the attempts to detect the formyl radical formed in the reaction among 3a, methacrolein, and TEMPO were unsuccessful.

(26) Stern-Volmer quenching studies between a photocatalyst (PC$\mathbf{1}$ or eosin Y) and pyrazoline 1a were inconclusive due to the fluorescent properties of $1 \mathrm{a}\left(\lambda_{\mathrm{em}}=476 \mathrm{~nm}\right.$, and $\left.\lambda_{\mathrm{ex}}=420 \mathrm{~nm}\right)$. 